\title{
Skeletal effects and refeeding syndrome in anorexia nervosa
}

\author{
VĂDUVA Naomi ${ }^{1}$, PUSKAS Lehel $^{1}$, RĂCHIȘAN Andreea-Liana ${ }^{1}$, TOMȘA Anamaria Magdalena ${ }^{1}$, \\ ALDEA Andreea Alexandra ${ }^{1}$, PERNE Mirela-Georgiana ${ }^{2}$
}

Corresponding author: Anamaria M. Tomşa, e-mail: anam.wf@gmail.com

${ }^{1}$ Department 9 - Mother \& Child, $2^{\text {nd }}$ Clinic of Pediatrics, „Iuliu Haţieganu” University of Medicine \& Pharmacy Cluj-Napoca, Romania ${ }^{2}$ Department 5 - Internal Medicine, "Iuliu Hatieganu” University of Medicine \& Pharmacy Cluj-Napoca, Romania

\begin{abstract}
Purpose Anorexia nervosa (AN) is a psychiatric disorder which can lead to numerous medical complications. In the clinical practice, during weight restoration, it is relatively common to observe potentially fatal complications, such as refeeding syndrome (RS). The objective of this case report is to highlight some key factors regarding nutritional support and the therapeutic approach in AN to avoid life-threatening complications, for example severe hypophosphatemia.

Methods We present the case of a 14-year-old girl suffering from AN, who was admitted to our hospital with lethargy, emaciation with bitemporal wasting, hirsutism and pitting lower extremity edema.

Results Based on the laboratory findings, the concerning condition of the patient was attributed to hypophosphatemia (1,64 mg/dl) caused by RS.

Conclusions Refeeding syndrome is a potentially lethal condition in the case of AN patients. Hypophosphatemia, which is a result of the refeeding syndrome, is a relatively common complication of overly aggressive nutritional rehabilitation. Physicians who are involved in treating this condition, should be aware of this potentially life-threatening syndrome, and assess their therapeutic approach accordingly
\end{abstract}

Keywords: anorexia nervosa, hypophosphatemia, refeeding syndrome, nutritional support,

\section{Introduction}

Anorexia nervosa, one of the most lethal psychiatric disorders, is distinguished by severe restriction of calorie intake caused by intense fear of weight gain and distorted perception of body image, which results in low body weight - according to the DSM-5 (Diagnostic and Statistical Manual of Mental Disorders, $5^{\text {th }}$ edition). AN has one of the highest mortality rates of any mental illness due to the medical complications of low weight and also aggressive nutritional rehabilitation can be potentially fatal, due to the refeeding syndrome (RS) $(1,2)$. The main risk factors for developing medical complications in AN are the degree of weight loss and the chronicity of this illness $(3-5,6)$. Many organs and systems are profoundly altered in severe anorexia such as: cardiovascular system, gynecological and reproductive one, endocrine system, gastrointestinal, neurological, and hematological system. Also, special attention should be paid to RS, that could occur because of fluid and electrolyte shifts during aggressive nutritional rehabilitation of patients with anorexia.

The RS is characterized by electrolyte disturbances, leading to clinical manifestations like congestive heart failure, rhabdomyolysis, hemolytic anemia, peripheral edema, delirium, seizures, coma and even sudden death (7-10). Refeeding hypophosphatemia $(\mathrm{RH})$ is the most common complication of nutritional restoration for patients with AN. The highest risk of hypophosphatemia seems to be in patients who weigh less than $70 \%$ of their ideal body weight or lose weight rapidly (11).

All anorexic patients should be very closely monitored during the first few weeks of the refeeding process. Restoring weight should be done avoiding very rapid increases in the daily caloric intake and quickly correcting electrolyte abnormalities.

\section{Case report}

A 14 year-old girl, without any remarkable psychiatric history or previously diagnosed co-morbidities, was admitted to our hospital for the inpatient treatment of her ongoing eating disorder. She reported a dramatic decline in her weight before her admission to the hospital, a total of 21 kilograms (from 50 to $29 \mathrm{~kg}$ ) in the last 9 months. The patient mentioned a correlation between experiencing excessive levels of stress and anxiety and her abnormal eating tendencies. Affirmatively, she voluntarily underwent these dietary restrictions in order to meet a distorted feminine body image, which she considered desirable. When asked about inducing vomiting, purging or doing excessive physical excercise, she denied doing so. Subsequently, the patient admitted missing her menstrual cycle for the last 5 months.

In the ER (emergency room), several vital signs were noted for their significance, such as a rectal temperature of $36,3^{\circ} \mathrm{C}$, heart rate of $50 \mathrm{BPM}$, a blood pressure of $90 / 50 \mathrm{mmHg}$, and respiratory rate of 14 . While performing the physical examination, we observed lethargy, emaciation with bitemporal wasting, hirsutism and pitting lower extremity edema. The remainder of her clinical examination was unremarkable (Figure 1-A, B, C).

Laboratory studies (Table 1) were notable for severe hypophosphatemia. A mild elevation of transaminases and mild augmentation of creatinine levels were also noted. Her hospital evolution was otherwise notable for steadily decreasing blood counts. During her hospitalization, she was 
found to be pancytopenic. She was placed briefly on reverse contact isolation for infection prophylaxis until her white count and ANC increased.

During her hospitalization, she benefited from the support of a pediatric psychiatrist, who offered psychological counseling and was present on rounds to discuss the current mental status and the correct therapeutical approach with the medical team.

Initially, the patient's daily meal plan's calorie content was set at $950 \mathrm{kcal} /$ day. With liquid supplementation and with the help of the medical team, assistants and the parents, this goal was consistently met. At first, the patient's body weight remained stationary. However, after the first few days of hospitalization, her weight began to increase consistently. The daily target calorie intake was raised by $200 \mathrm{kcals}$ on the $4^{\text {th }}$ day of admission, and by $350 \mathrm{kcals}$ on the $10^{\text {th }}$ day, to provide $1500 \mathrm{kcals} /$ day at the discharge. The decreased phosphorus levels were corrected progressively with the administration of phosphorus supplementation (Table 2).

In contrast with the radical improvement of the patient's physical condition, we observed increased levels of anxiety and non-compliance in her behavior, regarding the treatment and the process of renutrition. The distorted perception of body image and the temptation of abnormal eating behavior persisted. In order to overcome these psychological challenges, we initiated antipsychotic therapy with Risperidone, $0.25 \mathrm{mg} /$ day (at bedtime) initially, which later was increased to $2 * 0.25 \mathrm{mg} /$ day, with the gradual improvement of the distorted body image.

\section{Discussion}

Based on DSM-5, our patient was diagnosed as having anorexia nervosa, fulfilled all the criteria: 1-restriction of caloric intake that lead to a low body weight $(\mathrm{BMI}=11,48$ $\mathrm{kg} / \mathrm{m}^{2}$ ); 2-intense fear of becoming fat; 3-distorted perception of body shape and weight (1). AN is associated with general medical complications, which can occur in many organ systems and psychotic disturbances due to weight loss and malnutrition. She presented with significant co-morbidities like: bradycardia, electrolyte imbalance, BMI $<12 \mathrm{~kg} / \mathrm{m}^{2}$, severe depression with suicidal ideation. Even though a wellcalculated nutritional rehabilitation plan had been established, RS was developed. RS is defined as a clinical complication that can occur during aggressive nutritional rehabilitation of malnourished patients. It is a relatively common problem in patients with severe anorexia, 20-40\% of hospitalized patients are at risk of developing it (12). The main event of RS is the development of severe hypophosphatemia, leading to cardiovascular, respiratory, hematological, and neurological manifestations. Hypophosphatemia a predictable complication, thar mostly occur during the first 2 weeks of refeeding process. The best predictor for $\mathrm{RH}$ is the severity of the nadir body weight leading to a mortality about $30 \%$ (13) in this category of patients. However, RS is a preventable condition, some cases have been reported despite of a correct application of nutritional support. It can be avoided by restoring weight with an initial number of calories that is close to the resting energy expenditure, slowly increasing in daily caloric intake and closely monitoring the patient clinically and biochemically. If the RS occurs, like in our case, it should immediately slow nutritional replenishment and intravenously correct electrolyte abnormalities, especially hypophosphatemia, while evaluating the cardiovascular system. Our goals of nutritional rehabilitation were to restore weight, normalize eating patterns, achieve normal perceptions of hunger and satiety, and correct biological and psychological sequelae of malnutrition. The European Society of Clinical Nutrition and Metabolism guidelines (ESPN) recommend to start caloric repletion with $20 \mathrm{kcal} / \mathrm{kg} /$ day and gradually increase per week until the patient is metabolically stable, with a safe therapeutic goal to gain $0,5-1 \mathrm{~kg} /$ week (14). Within a month of treatment, most of the complications remitted, and her weight continued to increase slowly. Her caloric intake was liberalized to general guidance of three meals and three snacks per day. Some complications such as: amenorrhea, mild pericardial effusion and psychiatric disorders persisted for a long time.

This case may suggest that there is a limit at which point the medical complications may overweigh the benefits of weight gain. Moreover, achieving adequate compliance regarding the individual engagement in nutritional restoration and weight gain is made difficult by several obstacles. AN, being a psychiatric disorder, can't be treated as just a somatic disease. The mental health impairment must also be taken into consideration and treated accordingly. In our case, even if patient's vital signs were stabilized and weight started to increase, abnormal eating behavior persisted. As she started to recover and felt her body getting larger, her anxious and depressive thoughts were intensified, becoming more irritable, and sometimes describing suicidal ideas.

It is known in literature that psychotic episodes occur in 10$15 \%$ of eating-disordered patients (15). Malnutrition can contribute to psychosis through many pathways. Specific nutritional deficiencies including thiamine, niacin, and vitamin B12 together with sleep deprivation and metabolic derangements are main medical causes of psychosis. After weight restoration, patients with $\mathrm{AN}$ are likely to have resolution of psychosis symptoms, but antipsychotic medications should not be neglected to get an optimal and persistent response. Consequently, nutrition restoration is a core element in treatment because of the need to restore weight to avoid severe physical complications and to improve cognitive function to make psychological interventions useful and effective. After a good nutritional rehabilitation, the patient was transferred to the psychiatric unit for the second phase of the treatment, which is focused on normalization of eating behavior followed by relapse prevention. Psychological intervention includes supportive therapy, behavioral intervention, initial assessment for patient's insight, motivation for recovery and resolving family conflicts. In addition, psychoeducation as well as family involvement and support are crucial to help the patient to progress. Behavioral intervention is necessary to improve eating habits and the negative perception of body 
image. Individuals with AN can recover. However, they're at increased risk to drop out from treatment programs and relapse during periods of high stress or during triggering situations. Ongoing therapy or periodic appointments during times of stress may help them to remain healthy $(16,17)$.

In conclusion, refeeding syndrome is a potentially lethal condition in AN patients. Herein, we described a case severe hypophosphatemia occurred during the refeeding process. We emphasize the fact that one should 'start low and go slow' in the management of refeeding in anorexia nervosa patients.

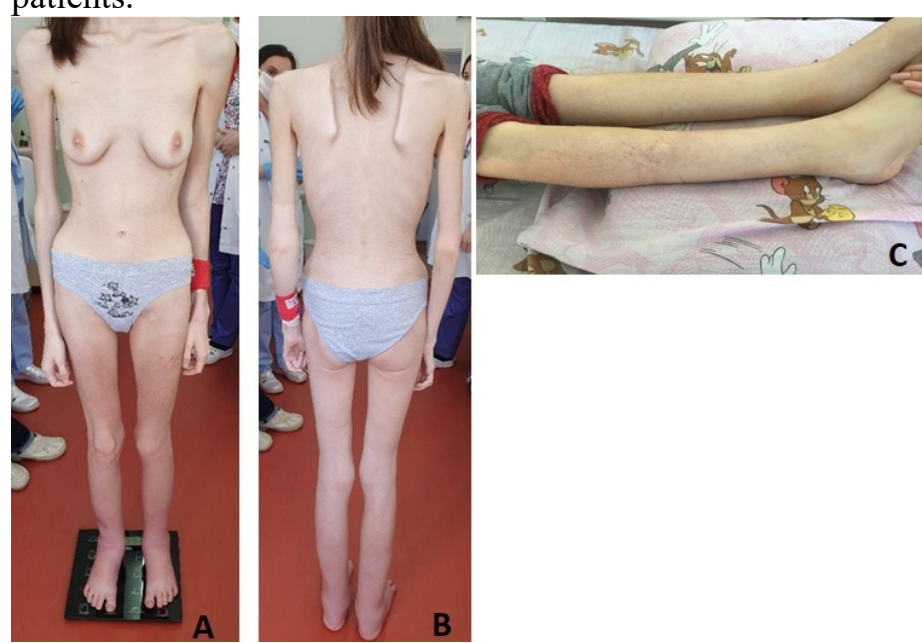

Figure 1-A, B, C: Physical examination at the time of hospital admission

Table 1. Important laboratory results from admission to discharge

\begin{tabular}{|l|l|l|l|}
\hline & On Admission & Day 10 & Discharge \\
\hline $\begin{array}{l}\text { Sodium (137-145 } \\
\text { mmol/l) }\end{array}$ & 138 & 142 & 140 \\
\hline $\begin{array}{l}\text { Potassium (3.5-5.1 } \\
\text { mmol/l) }\end{array}$ & 4.3 & 4.1 & 4.7 \\
\hline $\begin{array}{l}\text { Phosphorus (2.5-4.5 } \\
\text { mg/dl) }\end{array}$ & 1.64 & 2.77 & 4.34 \\
\hline $\begin{array}{l}\text { Creatinine (0.52-1.04 } \\
\text { mg/dl) }\end{array}$ & 1.06 & 0.51 & 0.39 \\
\hline $\begin{array}{l}\text { AST (15-46 u/l) } \\
\text { ALT (13-69 u/l) }\end{array}$ & 50 & 33 & 23 \\
\hline $\begin{array}{l}\text { Hemoglobin (12-16 } \\
\text { g/dl) }\end{array}$ & 13.1 & 57 & 28 \\
\hline $\begin{array}{l}\text { WBC count (4.5-10.8 } \\
\text { K/ul) }\end{array}$ & 7.93 & 3.56 & 5.89 \\
\hline $\begin{array}{l}\text { Platelets (150-450 } \\
\text { K/ul) }\end{array}$ & 116 & 125 & 233 \\
\hline
\end{tabular}

Table 2. Treatment course by day of hospitalization

\begin{tabular}{|l|l|l|l|l|}
\hline $\begin{array}{l}\text { Day of } \\
\text { hospital } \\
\text { admission }\end{array}$ & $\begin{array}{l}\text { Weight } \\
(\mathrm{kg})\end{array}$ & $\begin{array}{l}\text { Prescribed } \\
\text { meal plans } \\
(\mathrm{kcals})\end{array}$ & $\begin{array}{l}\text { Phosphorus } \\
\text { level } \\
(2.7-4.6 \\
\mathrm{mg} / \mathrm{dL})\end{array}$ & $\begin{array}{l}\text { Phosphorus } \\
\text { supplementation } \\
(\mathrm{mmol})\end{array}$ \\
\hline 1 & 29.4 & 950 & 1.64 & 2.35 \\
\hline 7 & 31.5 & 1150 & 1.28 & 2.52 \\
\hline 10 & 32.4 & 1500 & 2.77 & 2.60 \\
\hline Discharge & 33.9 & 1800 & 4.24 & 2.71 \\
\hline
\end{tabular}

\section{References:}

1. Brown C, Mehler PS (2015) Medical complications of anorexia nervosa and their treatments: an update on some critical aspects. Eat Weight Disord. 20(4):419-25.

2. Gunarathne T, McKay R, Pillans L, McKinlay A, Crockett P (2010) Refeeding syndrome in a patient with anorexia nervosa. BMJ 340:c56.

3. Whitelaw M, Lee KJ, Gilbertson H, Sawyer SM (2018) Predictors of Complications in Anorexia Nervosa and Atypical Anorexia Nervosa: Degree of Underweight or Extent and Recency of Weight Loss? J Adolesc Heal. 63(6):717-723.

4. Milaciu M, Ciumărnean L, Sampelean D, Negrean V, Milaciu C, Acalovschi (2018). Non-cardiometabolic comorbidities of non-alcoholic fatty liver disease. Balneo Res J 9: 43-49.

5. Maierean A, Ciumărnean L, Alexescu $\mathrm{T}$, Domokos $\mathrm{B}$, Râjnoveanu A, Arghir O, Todea D, Buzoianu AD, Dogaru G, Bordea R (2019). Complementary therapeutic approaches in asthma. Balneo Res J 10: 204-212.

6. Picos A, Rachisan AL, Dadarlat A (2018). Minimally Invasive Dental Treatment Using Composites and Ceramics in GERD Diagnoses Patients. Mater Plast 55(2):252-54.

7. Castro MA, Martinez CV (2018) The refeeding syndrome Importance of phosphorus. Medicina Clinica 150(12):472478.

8. Mehler PS, Winkelman AB, Andersen DM, Gaudiani JL (2010) Nutritional rehabilitation: practical guidelines for refeeding the anorectic patient. J Nutr Metab 625782.

9. Rachisan AL, Gheban D, Miu N (2013). Cutaneous sarcoidlike granulomas in a child known with Nijmegen Breakage Syndrome. Iran J Pediatrics 23(1):100-104.

10. Rachisan AL, Hrusca A, Gheban D, Cainap S, Pop TL, Baican A, Fodor L , Miu N, Andreica M (2012). Granulomatous cheilitis of Miescher - the diagnostic proof for a Melkersson Rosenthal. Rom J Morphol Embriol 53(3):851-3.

11. Kells M, Gregas M, Weeder K (2019) Factors Associated With Refeeding Hypophosphatemia (RH) In Hospitalized Adolescents And Young Adults (AYA) With Anorexia Nervosa (AN). J Adolesc Heal 64: S50-S51.

12. Davies JE, Cockfield A, Brown A, Corr J, Smith D, Munro C (2017) The medical risks of severe anorexia nervosa during initial re-feeding and medical stabilisation. Clin Nutr ESPEN 17:92-99.

13. Brown CA, Sabel AL, Gaudiani JL, Mehler PS (2015) Predictors of hypophosphatemia during refeeding of patients with severe anorexia nervosa. Int J Eat Disord. 48(7):898-904.

14. Vamshidhar R, Myrta DV (2015) Abnormal Liver Function Tests in an Anorexia Nervosa Patient and an Atypical Manifestation of Refeeding Syndrome. Case Rep Gastroenterol 9: 261-265.

15. Singer P, Blaser AR, Berger MM, Alhazzani W, Calder PC, Casaer MP, et al (2019) ESPEN guideline on clinical nutrition in the intensive care unit. Clin Nutr. 38:48-79.

16. Tomsa AM, Alexa AL, Junie ML, Rachisan AL, Ciumarnean L (2019). Oxidative stress as a potential target in acute kidney injury. PEERJ e8046.

17. Balan C, Chis MI, Rachisan AL, Baia M (2018). A vibrational study of inulin by means of experimental and theoretical methods. J Mol Struct. 1164:84-88. 